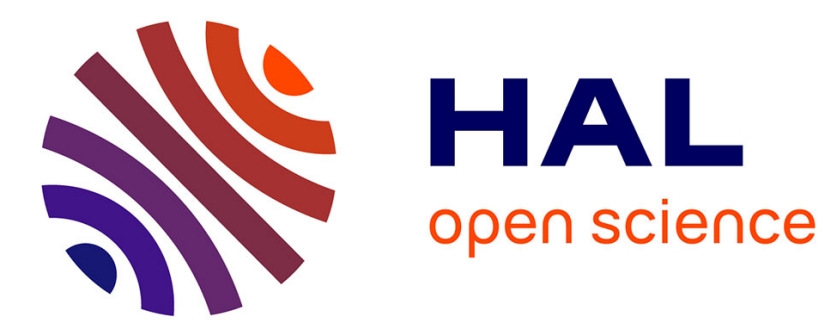

\title{
Dépérissement forestier le long du littoral tunisien sous l'effet des embruns marins pollués.
}

Naceur El Ayeb, Mustapha Ksontini, Belgacem Henchi, Jean-Pierre Garrec

\section{To cite this version:}

Naceur El Ayeb, Mustapha Ksontini, Belgacem Henchi, Jean-Pierre Garrec. Dépérissement forestier le long du littoral tunisien sous l'effet des embruns marins pollués.. Revue forestière française, 2004, 56 (3), pp.213-218. 10.4267/2042/5093 . hal-03449420

\author{
HAL Id: hal-03449420 \\ https://hal.science/hal-03449420
}

Submitted on 25 Nov 2021

HAL is a multi-disciplinary open access archive for the deposit and dissemination of scientific research documents, whether they are published or not. The documents may come from teaching and research institutions in France or abroad, or from public or private research centers.
L'archive ouverte pluridisciplinaire HAL, est destinée au dépôt et à la diffusion de documents scientifiques de niveau recherche, publiés ou non, émanant des établissements d'enseignement et de recherche français ou étrangers, des laboratoires publics ou privés. 


\title{
RISOUES NATURELS OU HUMAINS
}

\section{DÉPÉRISSEMENT FORESTIER \\ LE LONG DU LITTORAL TUNISIEN \\ SOUS L'EFFET DES EMBRUNS MARINS POLLUÉS}

\author{
Naceur El Ayeb - Mustapha Ksontini - Belgacem Henchi - Jean-Pierre Garrec
}

Le dépérissement des végétaux en bordure du littoral, constaté durant les années 1970 en Tunisie (Sethom, 1977), présente, depuis le début des années 1980, une évolution préoccupante (Garrec, 1994). Un phénomène identique a été signalé sur le littoral méditerranéen français aux abords de grands centres industrialo-portuaires (Devèze et Sigoillot, 1978) et en Italie à l'embouchure de fleuves côtiers très pollués (Gellini et al., 1981). Il a généralement été associé à la pollution de la surface de la mer par les détergents et les hydrocarbures.

\section{SUIVI SANITAIRE DES ARBRES}

La prospection de la région du Cap Bon a débuté au mois de mai 1996 et a été terminée en février 1998. La vigueur des arbres et arbrisseaux des peuplements littoraux a été étudiée. L'observation macroscopique a porté sur l'état sanitaire de l'arbre, sa biomasse, la couleur des feuilles, la localisation de nécroses. Les mêmes classes de dépérissement des arbres que celles appliquées dans le cadre du programme DEFORPA sur le dépérissement des forêts attribuable à la pollution atmosphérique (Garrec et al., 1989) ont été adoptées.

L'investigation a été effectuée sur des arbres adultes (10 individus), sur 20 placettes différentes le long du littoral méditerranéen au Cap Bon. Deux types de placettes ont été retenus pour les prélèvements. Elles sont localisées dans les régions de Oued El Bir et de Oued Ksob de Kelibia au Cap Bon (cf. figure 1, p. 215). Les prélèvements ont concerné des arbres sur des placettes dépérissantes situées en bordure de mer à moins de 50 mètres de la côte et directement sous l'influence des embruns marins (20 arbres). La classe de dépérissement de l'arbre est déterminée grâce à un document technique du groupe de travail des experts méditerranéens (Sanchez et al., 1994) qui correspond à la photographie de l'arbre avec son degré de dépérissement (5 classes). Pour chaque placette, les prélèvements ont concerné des branches prélevées dans le tiers supérieur du houppier. Pour caractériser le degré de sensibilité des espèces végétales (arbres et arbrisseaux) à ce phénomène, on a utilisé l'échelle suivante : I : espèce tolérante, II : espèce peu sensible, III : espèce sensible.

Des degrés de sensibilité différents ont été observés dans le comportement des espèces exposées aux embruns marins au littoral du Cap Bon (tableau I, p. 215).

Pour lutter contre l'effet nocif des embruns marins, les espèces les plus résistantes sont recommandées pour le reboisement aux abords immédiats du rivage (exemple : Acacia cyclopis) ; les espèces les moins résistantes, en retrait du rivage avec une légère protection (exemple : Acacia 
Naceur El Ayeb - Mustapha Ksontini - Belgacem Henchi - Jean-Pierre Garrec

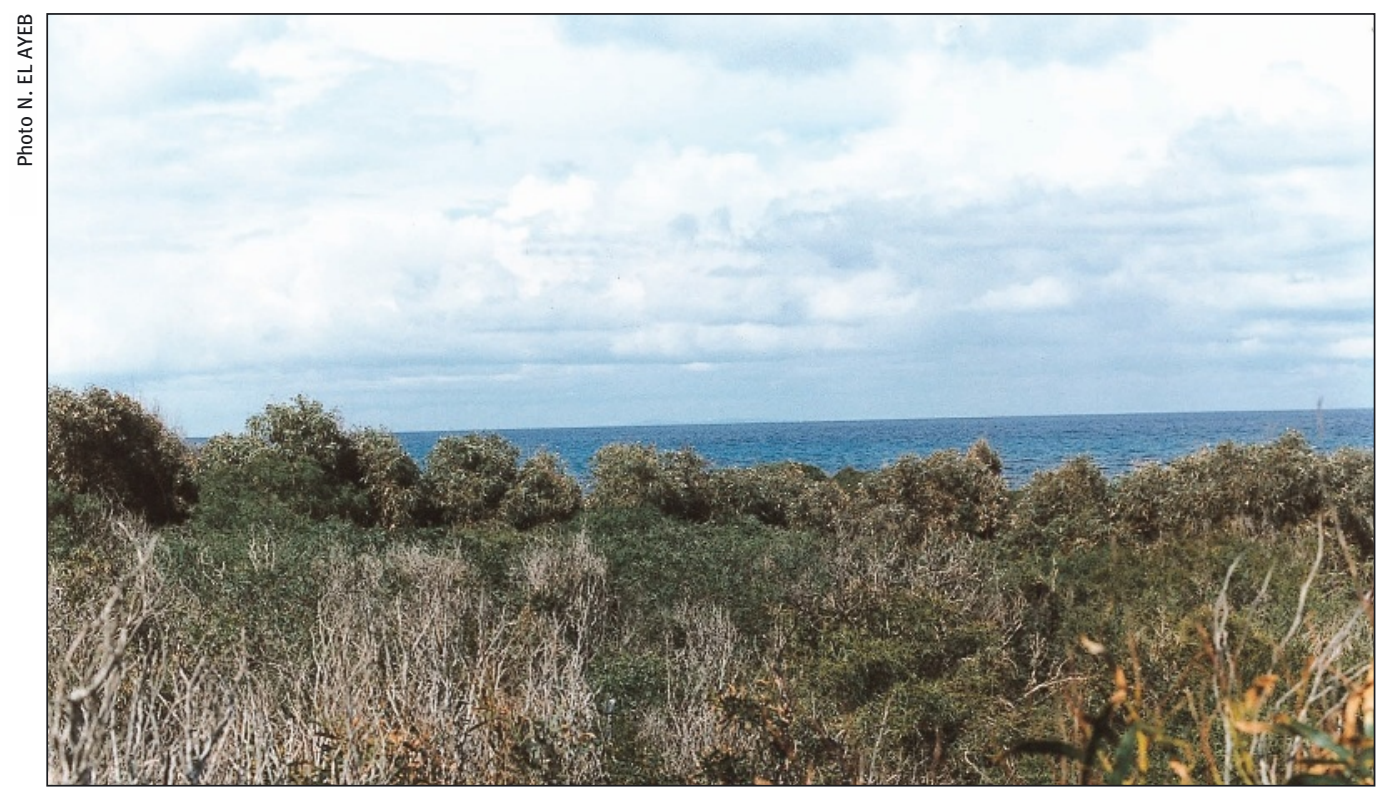

Photo 1

Vue d'ensemble de la forêt littorale Oued El Bir Acacia cyanophylla et Eucalyptus gomphocephala montrant des nécroses foliaires

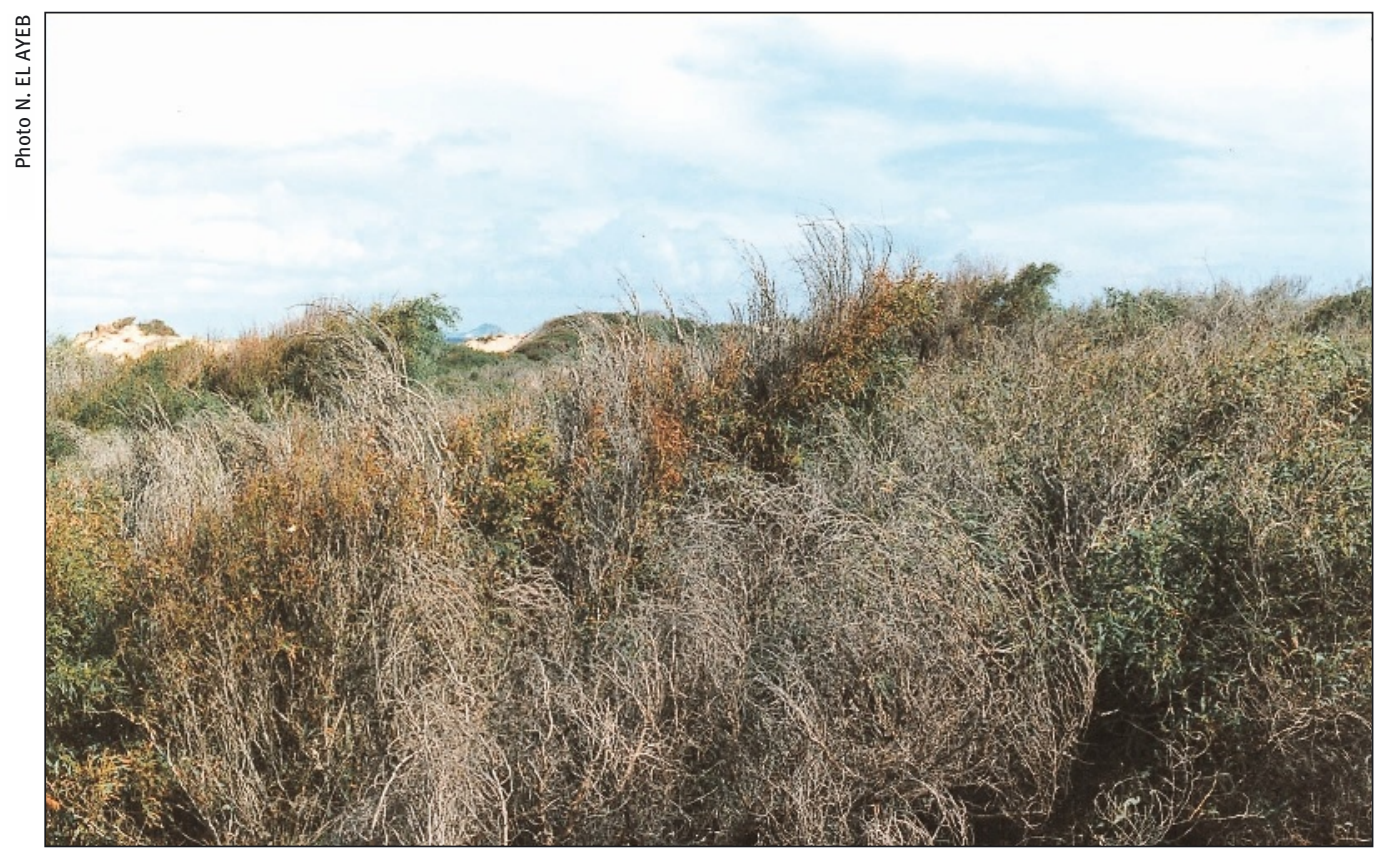

Photo 2

Acacia cyanophylla (station Oued El Bir) soumis à l'action des embruns marins pollués ; arbres à port buissonnant ; la face exposée au vent de mer dominant est entièrement défoliée 


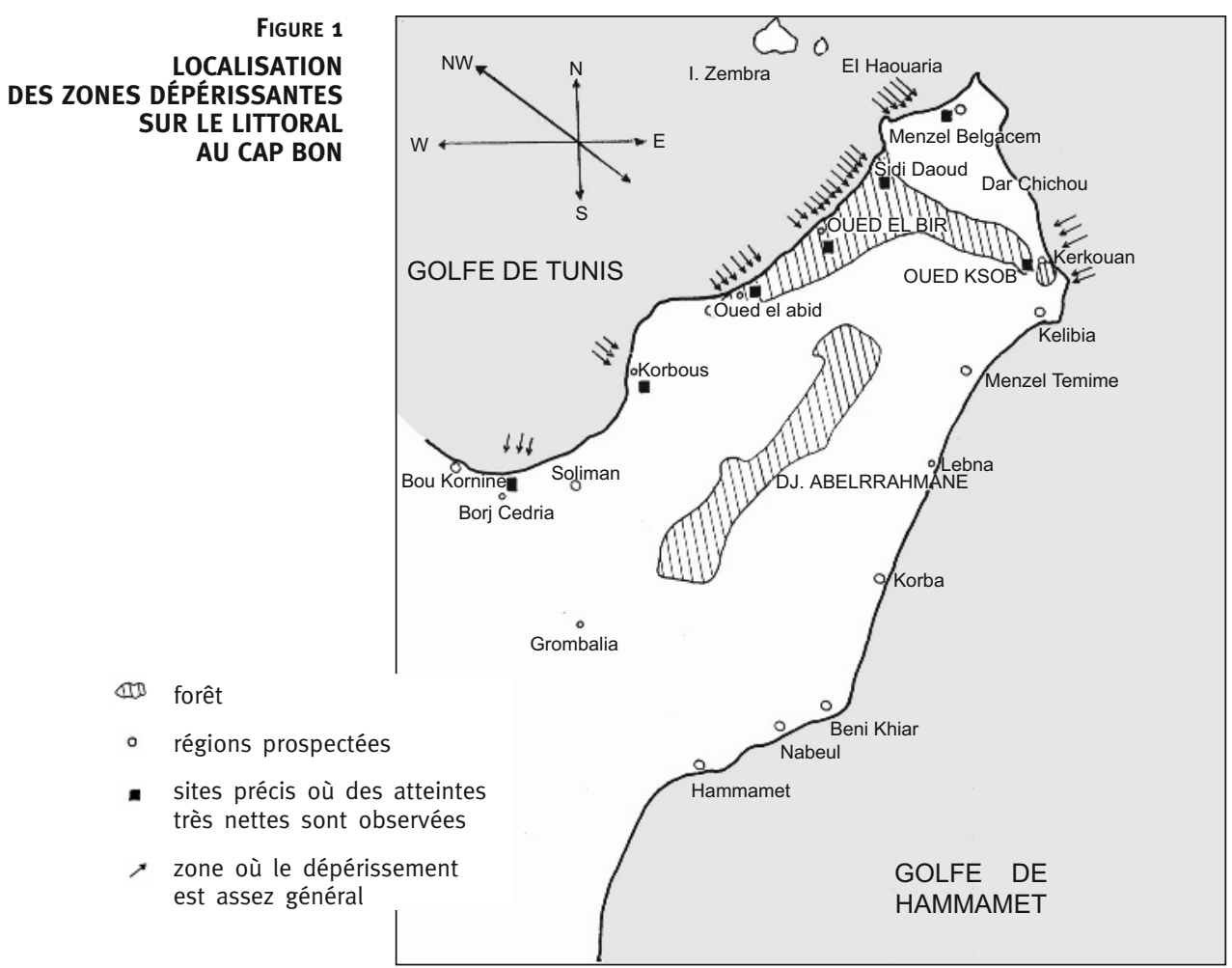

TABLEAU I

Sensibilitê relative indicative aux embruns marins pollués pour les arbres et arbrisseaux acclimatés en région méditerranéenne tunisienne

\begin{tabular}{|c|c|c|}
\hline Espèces tolérantes & Espèces peu sensibles & Espèces sensibles \\
\hline $\begin{array}{l}\text { Acacia cyclopis } \\
\text { Atriplex halimus } \\
\text { Ceratonia siliqua } \\
\text { Chamerops humilis } \\
\text { Cistus monspeliensis } \\
\text { Erica multiflora } \\
\text { Juniperus oxycedrus } \\
\text { Juniperus phoenicea } \\
\text { Pistacia lentiscus } \\
\text { Retama raetam } \\
\text { Rosmarinus officinalis } \\
\text { Stipa tenacissima } \\
\text { Tamarix gallica } \\
\text { Ziziphus lotus }\end{array}$ & $\begin{array}{l}\text { Acacia cyanophylla (saligna) } \\
\text { Acacia floribunda } \\
\text { Amygdalis communis } \\
\text { Asparagus albus } \\
\text { Calicotome villosa } \\
\text { Casuarina glauca } \\
\text { Eucalyptus leucoxylon } \\
\text { Ficus carica } \\
\text { Halimium halimifolium } \\
\text { Olea europaea } \\
\text { Opuntia ficus-barbarica } \\
\text { Phillyrea angustifolia } \\
\text { Phoenix dactylifera } \\
\text { Pinus canariensis } \\
\text { Pinus halepensis } \\
\text { Pinus pinea } \\
\text { Prunus domestica } \\
\text { Punica granatum } \\
\text { Quercus coccifera } \\
\text { Quercus ilex } \\
\text { Smilax aspera }\end{array}$ & $\begin{array}{l}\text { Cupressus sempervirens } \\
\text { Eucalyptus camaldulensis } \\
\text { Eucalyptus gomphocephala } \\
\text { Eucalyptus lehmanii } \\
\text { Eucalyptus occidentalis } \\
\text { Eucalyptus torquata }\end{array}$ \\
\hline
\end{tabular}


Naceur El Ayeb - Mustapha Ksontini - Belgacem Henchi - Jean-Pierre Garrec

cyanophylla) et les espèces sensibles, loin du rivage avec une bonne protection (exemple : Eucalyptus gomphocephala) (El Ayeb et al., 2000).

\section{UNE RÉPARTITION IRRÉGULIĖRE DES ZONES DÉPÉRISSANTES, LIÉE AU VENT}

Des atteintes sont actuellement visibles en de très nombreux points du littoral méditerranéen en Tunisie. Les indications fournies sur la figure 1 (p. 215) ont trait aux principales zones où des dépérissements ont été enregistrés au Cap Bon. Cette carte n'a qu'une valeur indicative et ne prétend pas à l'exhaustivité (El Ayeb et al., 1996). Le phénomène touche, à des degrés divers, toutes les espèces du maquis méditerranéen du littoral tunisien du Cap Bon. Le dépérissement est loin d'être uniforme. Les zones où la côte est tourmentée et accidentée sont moins atteintes que les zones de côtes basses, largement ouvertes aux vents de mer. Le vent dominant s'observe sur le côté occidental du Cap Bon (direction Nord et Nord-Ouest) (Garrec et El Ayeb, 2001). Les surfaces des zones affectées sont très irrégulières le long du littoral, et généralement elles concernent une ceinture de 1 à 2 kilomètres dans les terres loin de la ligne du rivage et ceci en fonction de la vitesse du vent dominant (El Ayeb, 1999).

\section{SYMPTOMATOLOGIE DU DÉPÉRISSEMENT}

La photographie 1 (p. 214) montre l'aspect d'un peuplement d'Acacia cyanophylla et d'Eucalyptus gomphocephala plantés dans la forêt domaniale Menzel Belgacem, à proximité immédiate de la mer. Les branches et les rameaux des premiers arbres directement soumis au contact des embruns présentent une défoliation complète. Les branches et les rameaux de deuxième ordre, plus en retrait, bénéficient d'un effet écran, la défoliation est marquée mais incomplète et des anomalies de coloration du feuillage sont visibles. Les arbres, à l'abri des deux premières lignes, conservent un aspect presque normal avec peu ou pas de symptômes de dépérissement. Les deux premières lignes d'arbres ont été rattachées à la classe 3 de dépérissement $(25 \%-60 \%$ de défoliation) et la troisième, à l'abri des deux autres, à la classe 2 de dépérissement (10 \%-25\% de défoliation). Au fur et à mesure de la mort des rameaux ou des individus les plus proches du littoral, le dépérissement atteint la végétation située à l'intérieur des terres (photo 2, p. 214). En général, les symptômes de dépérissement sont des nécroses de la bordure des feuilles puis du limbe foliaire pour les feuillus et de la partie apicale de l'aiguille pour les conifères (El Ayeb, 1998). Les nécroses marginales pourraient venir d'un dessèchement des feuilles mais la moitié de l'arbre endommagé est toujours du côté de la mer, ce qui exclut l'hypothèse d'une sécheresse atmosphérique ou d'un fort vent sec (sirocco) comme origine à ce phénomène (Rejeb et El Ayeb, 2000).

\section{CONCLUSIONS}

Il a été mis en évidence localement sur la côte méditerranéenne en Tunisie des symptômes de dépérissement sur l'ensemble de la végétation littorale. Les principales caractéristiques des symptômes observés in situ sur Acacia cyanophylla et Eucalyptus gomphocephala ont été définies. La symptomatologie des dommages et leur succession ont été caractérisées. Ces observations nous permettent de formuler des hypothèses sur la nature de l'agent desséchant et sur ses caractéristiques : il s'agit d'un agent de nature abiotique qui provient de la mer et est transporté 
par les vents vers la terre avec les aérosols marins ; il s'agit probablement d'un composé se trouvant dans la couche superficielle de l'eau, où se forment les aérosols. Les substances qui répondent le plus à ces conditions sont les tensioactifs et les hydrocarbures (El Ayeb, 2001). D’autres enquêtes (stimulation du phénomène de dépérissement au laboratoire par la même solution d'eau de mer polluée...) nous ont conduit à retenir l'hypothèse des tensioactifs (El Ayeb et al., 2004). Ces tensioactifs, après avoir attaqué les surfaces foliaires, facilitent la pénétration du sel dans les tissus, et ce sel serait la cause majeure des perturbations observées. Le fait que l'ensemble des espèces végétales du littoral soit affecté indique que ces dommages ne sont pas en relation avec des attaques de parasites ou de pathogènes. Il est important de souligner que le dépérissement des arbres en bordure du littoral n'est jamais dû à un facteur unique, mais à la conjonction d'un ensemble de phénomènes défavorables pour la croissance de l'arbre, tels que la sécheresse, les tempêtes, les problèmes de nutrition, qui conduisent à un affaiblissement général de l'arbre, et éventuellement à sa mort. Les moyens de lutte contre les effets des embruns marins sont aujourd'hui limités. Il faudrait, d'une part, diminuer la source de pollution qui constitue les détergents (produits lessiviels, etc.) de plus en plus utilisés en Tunisie et plus ou moins biodégradables ; d'autre part, augmenter le nombre de stations d'épurations et leur efficacité. L'inventaire des espèces forestières résistantes aux embruns marins et qui pourraient être utilisées pour le reboisement sur le littoral est une des solutions pour limiter ce phénomène de dépérissement.

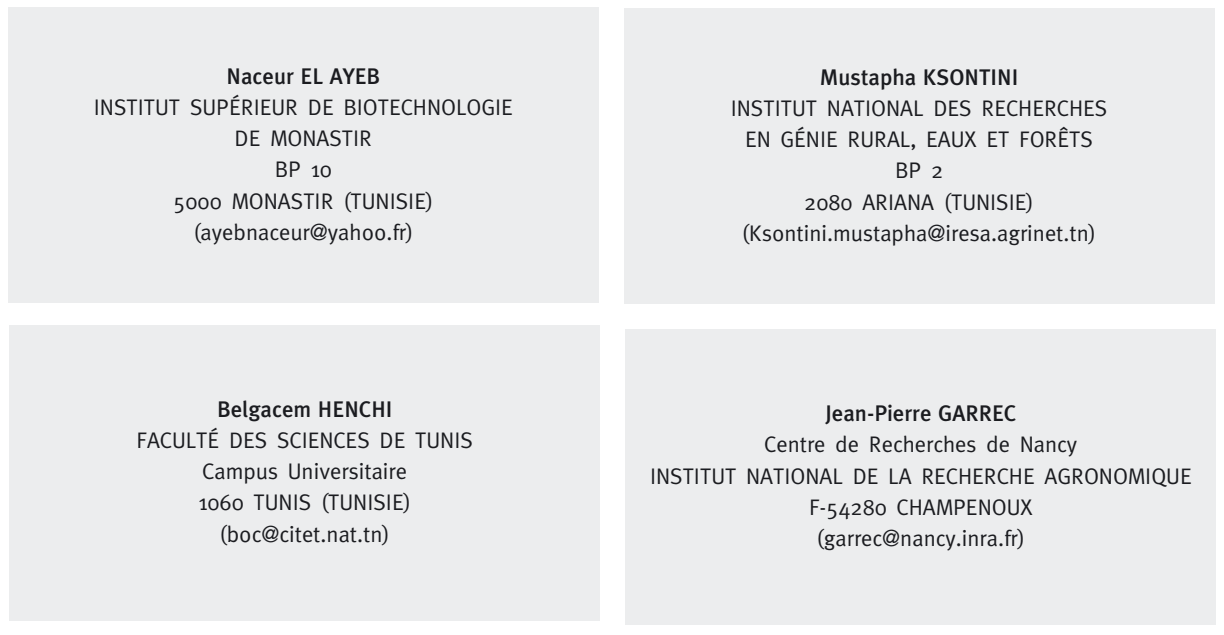

\section{BIBLIOGRAPHIE}

DEVEZE (L.), SIGOILLOT (J.-C.). - Les Arbres malades de la mer. - Eau, Aménagement, n 19, 1978, pp. $13-24$. EL AYEB (N.). - Dépérissement forestier au littoral par les embruns marins pollués. - Université Tunis El Manar, 2001. - 145 p. (Thèse de Doctorat).

EL AYEB (N.). - De quoi notre forêt côtière est-elle malade ? - Science plus, $\mathrm{n}^{\circ}$ 12(1), juillet 1998, p. 29.

EL AYEB (N.). - Pollution atmosphérique de la végétation littorale par les embruns marins au Cap Bon. In : II Colloque international "Sciences et Environnement", Bizerte (Tunisie), 3-5 décembre 1999, p. 72.

EL AYEB (N.), HENCHI (B.), GARREC (J.-P.), REJEB (M.N.). - Effets des embruns marins pollués sur les feuilles d'Acacia cyanophylla Lindl. et d'Eucalyptus gomphocephala Dc. du littoral tunisien. - Annals of Forest Science, vol. 61, 2004, pp. 1-9. 
Naceur El Ayeb - Mustapha Ksontini - Belgacem Henchi - Jean-Pierre Garrec

EL AYEB (N.), HENCHI (B.), REJEB (M.N.). - Dépérissement des arbres sur le littoral tunisien au Cap Bon. In : IX ${ }^{\text {es }}$ Journées nationales de Biologie, Monastir (Tunisie), 7-10 novembre 1996, p. 46.

EL AYEB (N.), REJEB (M.N.), HENCHI (B.), KSONTINI (M.). - Comportement des arbres forestiers sous l'effet de stress environnementaux, pollution locale par les embruns marins. Rapports sur le Projet national mobilisateur P97 BIRD 20. - Tunis : SERST ; INRGREF, 2000. - 25 p.

GARREC (J.-P.). - Les Dépérissements littoraux d'arbres forestiers. - Revue forestière française, vol. XLVI, $n^{\circ} 5$, 1994, pp. 454-457.

GARREC (J.-P.), EL AYEB (N.). - Il problema degli aerosol marini inquinati in Francia e Tunisia. Danni alla vegetazione costiera ed inquinamento da tensioattivi. - Linea ecologica, vol. 33, $n^{\circ}$ 1, 2001, pp. 51-54.

GARREC (J.-P.), RICHARDIN (I.), LE MAOUT (L.), ROSE (C.), GÉRARD (B.), MATHIEU (Y.). - Étude du pH et du pouvoir tampon des aiguilles de Sapins (Abies alba L.) et d’Épicéas (Picea abies L.) dépérissants. Application en symptomatologie ? - Annales des Sciences forestières, vol. 46, 1989, pp. 55-69.

GELLINI (R.), PANTANI (F.), BUSSOTTI (F.), RACANELLI (E.). - Sulla degradazione della vegetazione litoranea nella tenuta presidenziale di San Rossore. - Inquinamento, vol. 10, $\mathrm{n}^{\circ}$ 23, 1981, pp. 27-30.

REJEB (M.N.), EL AYEB (N.). - Origine du dépérissement forestier sur le littoral au Cap Bon : implication des embruns pollués. - Atelier d'évaluation, IRESA, Ministère de l'Agronomie, PF : Écologie, sélection des espèces et reboisement, 2000. - $12 \mathrm{p}$.

SANCHEZ PENA (G.), ECONOMOU (A.), BECCU (E.), CANU (G.), COCCO (S.), BUSSOTTI (F.), CENNI (E.), COZZI (A.), CONCEIÇAO ANDRADA (M.). - Essences forestières méditerranéennes : évaluation de l'état des houppiers. - Commission des Communautés européennes, 1994. - 78 p.

SETHOM (H.). - L'Agriculture de la presqu'île du Cap Bon (Tunisie). Structures sociales et économie rurale. - Publications de l'Université de Tunis. Faculté des Lettres et Sciences humaines de Tunis. II Série : Géographie, vol. V, 1977.

\section{DÉPÉRISSEmENT FORESTIER LE LONG DU LITTORAL TUNISIEN SOUS L'EFFET dES EmbRUNS MARINS POLLUÉS [Résumé]}

La végétation côtière du Cap Bon (Tunisie) a présenté, à partir des années 1980, un dépérissement. Notre travail en décrit les symptômes, présente l'ampleur du phénomène en Tunisie et classe certaines essences acclimatées selon leur sensibilité aux embruns marins. Les branches et les rameaux d'Acacia cyanophylla Lindl. et d'Eucalyptus gomphocephala Dc. qui sont exposés aux embruns marins présentent des symptômes caractéristiques qui ne sont pas imputables à des attaques parasitaires ou à l'effet unique du chlorure de sodium. Nous suggérons que ce phénomène de dépérissement est causé par les détergents domestiques qui se retrouvent sous forme concentrée dans les embruns.

\section{FOREST DIEBACK RLONG THE TUNISIRN COAST UNDER THE INFLUENCE OF POLLUTED SER SPRAY [Abstract]}

The coastal vegetation at Cap Bon (Tunisia) began to decay in the 8os. Our work describes the symptoms, the extent of the phenomenon in Tunisia and classifies a number of acclimatised species according to their sensitivity to sea spray. Acacia cyanophylla Lindl. and Eucalyptus gomphocephala Dc. branches and twigs which are exposed to sea spray exhibit characteristic symptoms that are not attributable to parasite attacks or to solely to sodium chloride. We suggest that domestic detergents that are found in concentrated form in spray cause this dieback. 